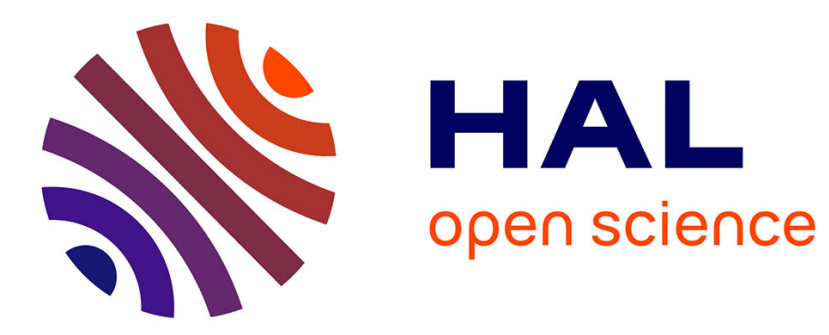

\title{
Third-order Riemann Pulses in Optical Fiber
}

\author{
Domenico Bongiovanni, Zhili Li, Benjamin Wetzel, Yi Hu, Stefan Wabnitz, \\ Roberto Morandotti, Zhigang Chen
}

\section{To cite this version:}

Domenico Bongiovanni, Zhili Li, Benjamin Wetzel, Yi Hu, Stefan Wabnitz, et al.. Third-order Riemann Pulses in Optical Fiber. CLEO: Applications and Technology, May 2020, Washington, United States. pp.JTh2E.18, 10.1364/CLEO_AT.2020.JTh2E.18 . hal-03011048

\section{HAL Id: hal-03011048 \\ https://hal.science/hal-03011048}

Submitted on 18 Nov 2020

HAL is a multi-disciplinary open access archive for the deposit and dissemination of scientific research documents, whether they are published or not. The documents may come from teaching and research institutions in France or abroad, or from public or private research centers.
L'archive ouverte pluridisciplinaire HAL, est destinée au dépôt et à la diffusion de documents scientifiques de niveau recherche, publiés ou non, émanant des établissements d'enseignement et de recherche français ou étrangers, des laboratoires publics ou privés. 


\title{
Third-order Riemann Pulses in Optical Fiber
}

\author{
Domenico Bongiovanni ${ }^{1,2}$, Zhili Li ${ }^{1}$, Benjamin Wetzel ${ }^{3}$, Yi Hu${ }^{1}$, Stefan Wabnitz ${ }^{4}$, Roberto Morandotti ${ }^{1,5}$, and \\ Zhigang Chen ${ }^{1,6}$ \\ ${ }^{I}$ TEDA Applied Physics Institute and School of Physics, Nankai University, Tianjin 300457, China \\ ${ }^{2} I N R S$-EMT, 1650 Blvd. Lionel-Boulet, Varennes, QC J3X 1S2, Canada \\ ${ }^{3}$ XLIM Research Institute, CNRS UMR 7252, Université de Limoges, Limoges 87060, France \\ ${ }^{4}$ DIET, Sapienza University of Rome, Via Eudossiana 18, Rome 00184, Italy \\ ${ }^{5}$ Institute of Fundamental and Frontier Sciences, University of Electronic Science and Technology of China, Chengdu 610054, China \\ ${ }^{6}$ Department of Physics \& Astronomy, San Francisco State University, San Francisco, CA 94132, USA \\ zgchen@nankai.edu.cn,morandotti@emt.inrs.ca
}

\begin{abstract}
We report on the generation of third-order Riemann pulses in nonlinear optical fiber, obtained by tailoring the initial pulse in presence of high-order dispersion and Kerr nonlinearity.

Analytical and numerical results show controllable pulse steepening and shock formation. $\odot 2020$ The Author(s) OCIS codes: (190.0190) Nonlinear optics; (070.7345) Wave propagation; (190.3270) Kerr effect
\end{abstract}

Simple Riemann waves (RWs), solutions of the Inviscid Burgers' Equation (IBE), are of fundamental importance to study shock formation in different physical frameworks beyond hydrodynamics [1]. Experimental demonstration and control of RW signatures have been recently reported in nonlinear optics, in both the temporal [2-5] and spatial [6-7] domains. Nevertheless, standard RWs are achieved under both week dispersion/diffraction and strong nonlinearity, and have been only demonstrated in the self-defocusing regime of the nonlinear Schrödinger equation (NLSE). High-order dispersive effects in fibers can affect the RW dynamics by significantly deteriorating their expected temporal profiles. Here, we propose a conceptually new class of optical RWs which can be implemented in nonlinear optical fibers, namely, third-order Riemann pulses (TORPs). Such RW packets arise from the interplay between high-order dispersion and nonlinearity, and can be realized by properly tailoring the phase profile of an input ultrashort light pulse.

Our analysis starts by considering the NLSE that describes pulse propagation in a nonlinear optical fiber with Kerr nonlinearity, considering also both second- and third-order dispersions (SOD and TOD), as follows:

$$
i \frac{\partial A}{\partial Z}-\frac{\ddot{\beta}^{2}}{2} \frac{\partial^{2} A}{\partial T^{2}}-\frac{i \bar{\beta}^{3}}{6} \frac{\partial^{3} A}{\partial T^{3}}+|A|^{2} A=0 .
$$

In Eq. (1), $A(T, Z)$ is the dimensionless electric field envelope, while $T=t / T_{0}$ and $Z=z / L_{\mathrm{NL}}$, are the normalized temporal and propagation coordinates, respectively. We scale the pulse duration by $T_{0}$, and its peak power by $P_{0}$. $\ddot{\beta}^{2}=\beta_{2} /\left(\gamma P_{0} T_{0}^{2}\right)$ and $\bar{\beta}^{3}=\beta_{3} /\left(\gamma P_{0} T_{0}^{3}\right)$ are the dimensionless SOD and TOD terms, where $\gamma=k_{0} n_{2}$ is the nonlinear Kerr coefficient, $k_{0}$ is the vacuum wavenumber, $n_{0}$ and $n_{2}$ are the linear and nonlinear refractive index, and $\beta_{2}$ and $\beta_{3}$ denote the SOD and TOD coefficients of the fiber. Arbitrary solutions to Eq. (1) can be found by expressing the pulse envelope in a polar form by means of the Madelung transformation:

$$
A(T, Z)=\sqrt{\rho(T, Z)} \exp \left[-\frac{i}{\bar{\beta}} \int_{-\infty}^{T} u\left(T^{\prime}, Z\right) d T^{\prime}\right],
$$

with $\rho(T, Z)$ and $u(T, Z)$ being real functions. If the condition $\bar{\beta}<<1$ is satisfied, Eq. (1) can be reduced to a semiclassical approximation of the NLSE, known as nonlinear shallow water equation (NSWE) [1]. In general, the NSWE is expressed as a system of two coupled equations, and is characterized by two Riemann invariant solutions. Among them, specific solutions, known as simple RWs, can be obtained by setting one of these two Riemann invariants to be a null value, thus reducing the NSWE system to one equation only. More importantly, the propagation dynamics in the nonlinear optical fiber as governed by Eq. (1) can be efficiently modeled by the IBE, whose solution is expressed in an implicit form as:

$$
\rho(T, Z)=\rho_{0}\left(T+\frac{\beta^{2}}{2 \bar{\beta}^{3}} Z-\frac{5}{2} \sqrt[3]{3 \rho(z, t)^{2}} \bar{\beta} Z\right),
$$

where $\rho_{0}$ is the initial pulse envelope and $\tilde{\beta}=\ddot{\beta} / \bar{\beta}^{2}$. 

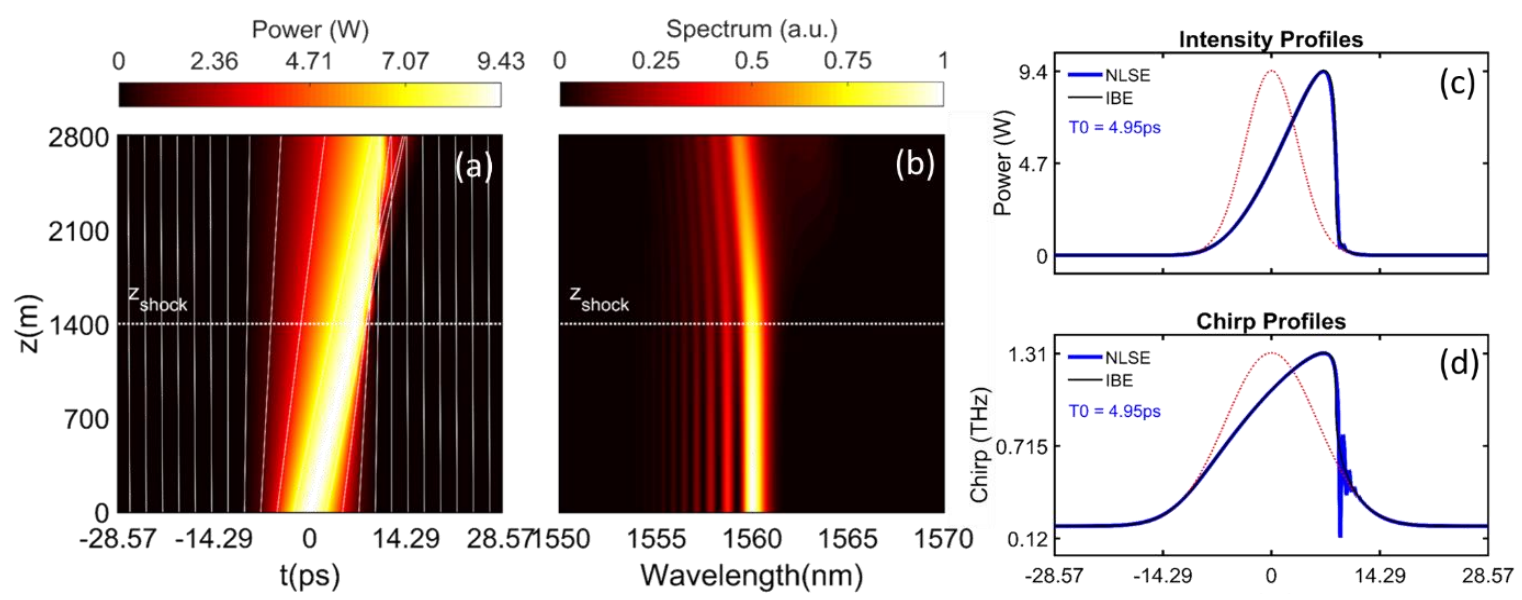

Figure 1: (a) Intensity and (b) spectral plots of NLSE simulations, modeling the evolution of a TORP over $2.8 \mathrm{~km}$ of dispersion-shifted fiber. The pulse intensity is compared with characteristic lines, obtained analytically from the IBE (white lines), showing shock formation at $\mathrm{z}=1.4 \mathrm{~km}$ (here characteristics lines intersect leading to a multi-valued solution (parameters: Gaussian pulse with 9.42W peak power and 8.24ps duration (FWHM) propagating in a DSF: $\beta_{2}=-0.154 \mathrm{ps}^{2} \cdot \mathrm{km}-1, \beta_{3}=0.1234 \mathrm{ps}^{3} \cdot \mathrm{km}-1$ and $\gamma=0.5 \mathrm{~W}-1 . \mathrm{km}-1$ at $\lambda_{0}=1550 \mathrm{~nm}$ ). Temporal (c) intensity and (d) chirp profiles are shown at the input and at shock point, comparing IBE predictions (solid black) with NLSE simulations (dashed blue).

From a physical viewpoint, the dynamics of a TORP are associated with a progressive steepening of the pulse envelope, and eventually the formation of a so-called gradient catastrophe (or shock wave formation), where the time derivative of the envelope tends to infinity. The distance at which the shock distance occurs can be analytically calculated by using the method of characteristics, that provides a parametric representation of the energy flow. Equation (3) represents the main result of this research work, mainly associated with the fact that the steepening evolution of the TORPs is uniquely managed by the TOD effect. As a consequence, TORPs exist both in the presence and in the absence of group velocity dispersion (GVD). Unlike standard RWs, already reported in the literature, here the contribution of the GVD only introduces a transversal shifting of the shock along the time axis, thus generating time-shifted RWs [5]. Remarkably, the sign of the GVD does not influence on the steepening process, which means that this new class of Riemann pulses can be also generated for negative values of $\beta_{2}$, i.e., in the self-focusing regime of the NLSE. From a technological perspective, TORPs can be synthesized by appropriately shaping the initial temporal optical pulse, so that the specific relationship between the instantaneous frequency chirp and the pulse amplitude remains valid in subsequent propagation [2]. To prove our theoretical analysis, we also performed NLSE simulations for a specific case of TORPs, with a Gaussian intensity profile. Numerical results are illustrated in Fig. 1 and are obtained by using the split-step Fourier transform method applied to Eq. (1). The target Riemann profile is obtained by imposing the appropriate phase modulation to a transformlimited Gaussian pulse (8.24 ps FWHM, 9.42 W peak power), according to Eq. (2). During propagation, the prechirped Gaussian pulse undergoes a progressive steepening of its leading edge, by maintaining a constant peak intensity, and subsequently reaches a nearly vertical front appearing at the mid-point of the fiber, i.e., $z_{\text {shock }}=1.4 \mathrm{~km}$, at time $t_{\text {shock }}=8.42 \mathrm{ps}$. In the Fourier domain, the spectrum associated with the TORP displays an Airy-like shape, which exhibits a higher intense main lobe located about $\lambda=10 \mathrm{~nm}$ away from the carrier wavelength (up to the shock distance), as shown in Fig. 1(b). Furthermore, both the intensity and chirp profiles, retrieved from NLSE simulations at the shock distance, display a quasi-perfect matching with IBE predictions [black lines in Figs. 1(c,d)], which is further confirmed by the overlap between the intensity distribution and the IBE characteristic line [white lines in Fig. 1(a)]. Indeed, characteristic lines intersect at the predicted shock distance, to give rise to a multi-valued solution.

This work opens the possibility to achieve an effective control of Riemann pulse dynamics in optical fibers, as currently explored in our experiment. Our finding may prove applicable for other physical systems, where an IBE approximation of the NLSE description provides a powerful tool for studying peculiar nonlinear wave dynamics.

\section{References}

[1] J. Bec and K. Khanin, "Burgers turbulence," Phys. Rep. 447, 1-66 (2007).

[2] B.Wetzel, et al., "Experimental generation of Riemann waves in optics: a route to shock wave control,” Phys. Rev. Lett. 117, 073902 (2016).

[3] S. Randoux, et al., “Optical random Riemann waves in integrable turbulence,” Phys Rev. Lett. 118, 233901 (2017).

[4] W. Wan, S. Jia, and J. W. Fleischer, "Dispersive superfluid-like shock waves in nonlinear optics,” Nature Phys. 3, 46 (2007).

[5] S. Wabnitz, “Optical tsunamis: shoaling of shallow water rogue waves in nonlinear fibers with normal dispersion,” J. Opt. 15, 064002 (2013).

[6] G. Marcucci, et al., “Optical spatial shock waves in nonlocal nonlinear media,” Adv. Phys. X 4, 1662733 (2019).

[7] D. Bongiovanni, et al., "Optical generation and control of spatial Riemann waves,” Opt. Lett. 44, 3542 (2019). 